\title{
Interdisziplinäre Teamarbeit auf einer Notfallstation
}

\author{
Hans Matter ${ }^{a}$, Basil Caduff, \\ Othmar Schöbc \\ a Ärztlicher Leiter Interdisziplinäre \\ Notfallstation, Gesundheits- \\ zentrum Zürich West \\ b Chefarzt Medizinische Klinik, \\ Spital Limmattal \\ c Chefarzt Chirurgische Klinik, \\ Spital Limmattal
}

\section{Zusammenfassung}

Der Betrieb einer Notfallstation mit fixen interdisziplinär arbeitenden notfalleigenen Teamleadern und einem ebenfalls interdisziplinär arbeitenden Assistentenpool stellt in der klinischen Notfallmedizin bei Erwachsenen in der Schweiz ein Novum dar. Ein in die tägliche Notfallarbeit voll integrierter Ärztlicher Leiter ist verantwortlich für die organisatorische und fachliche Führung der Notfallstation und vertritt ihre Interessen gegen innen und aussen. Die Abläufe in der Notfallstation werden optimiert, Ressourcen besser genutzt, kritische Situationen vermindert und die Kosten gesenkt. Durch die ständige Präsenz eines Kaderarztes, der alle Notfälle beurteilt, entsteht ein enormer Lerneffekt in der Assistentenausbildung, die Interdisziplinarität fördert ein flexibleres Denken und eine ganzheitlichere Beurteilung der Patienten. Der Betrieb einer «fast track»-Spur vermindert personelle und logistische Engpässe in Spitzenzeiten.

\section{Einführung}

Die Arbeit auf schweizerischen Notfallstationen ist bisher gekennzeichnet durch ein starres Dienstsystem sowohl auf Assistenten- wie auch auf Kaderarztebene. Unabhängig von der Gesamtbelastung der Notfallstation betreuen im Rahmen ihrer Ausbildung internistische Assistenten internistische Notfälle und chirurgische Assistenten chirurgische Notfälle. Eine fächerübergreifende und ganzheitliche Betreuung von Notfallpatienten ist nicht immer gewährleistet. Erschwerend kommt eine oft verzögerte oder fehlende Kaderarztpräsenz auf der Notfallstation hinzu, die durch andere Aufgaben bedingt ist. Daraus resultieren folgende Probleme:

- lebensbedrohliche Situationen werden oftmals durch einen unerfahrenen Assistenten primär beurteilt und behandelt;

Dr. Hans Matter FMH Innere Medizin

Interdisziplinäre Notfallstation Gesundheitszentrum Zürich West Urdorferstrasse 100 CH-8952 Schlieren Tel. 0447332546 Fax 0447332273
- unerfahrene Untersucher veranlassen unnötige (und mitunter auch falsche) Untersuchungen;

- es werden vermehrt unnötige Nachkontrollen im Spital statt bei den Hausärzten veranlasst;

- verzögertes Warten auf den Hintergrunddienst führt zu Unzufriedenheit bei Patienten und Personal;

- Ressourcen der Notfallstation werden nicht adäquat eingesetzt;

- ein fixer Ansprechpartner, der die Interessen der Notfallstation nach innen und aussen vertritt, fehlt.

\section{Interdisziplinäre Teamarbeit - ein neues} Konzept in der klinischen Notfallmedizin Das Gesundheitszentrum Zürich West in Schlieren ist ein Spital der erweiterten Grundversorgung mit 193 Akutbetten und einem Einzugsgebiet von etwa 90000 Einwohnern. 2005 behandelten wir 32732 ambulante, 2090 teilstationäre und 7391 stationäre Patienten. Das Gesundheitszentrum Zürich West umfasst die Disziplinen Chirurgie, Orthopädie, Innere Medizin (mit den Subspezialitäten Pneumologie, Kardiologie, Onkologie und Hämatologie), Urologie, Gynäkologie/Geburtshilfe, Radiologie und Anästhesiologie. Zusätzlich betreibt unser Spital eine anerkannte interdisziplinäre Intensivstation mit sechs Betten sowie einen Rettungsdienst.

Der Leistungsauftrag unserer Notfallstation umfasst die Erstversorgung und Betreuung aller stationären und nicht stationären Patienten inklusive Kinder sowie auch die teilweise Überwachung von intensivpflichtigen Patienten bei Engpässen auf der Intensivstation. Zur Verfügung stehen nebst einem Schockraum neun Behandlungsplätze, davon zwei sogenannte Kurzzeitbehandlungsspuren («fast track») sowie ein angegliedertes Ambulatorium mit zwei Zimmern und ein Gipszimmer.

Eine gutfunktionierende Notfallstation ist von strategischer Bedeutung für das Gesamtspital. Im Jahre 2004 beschloss deshalb die Spitalleitung einerseits die bauliche Erneuerung der 
Notfallstation, andererseits wurde erkannt, dass ohne grundlegende strukturelle und organisatorische Veränderungen auf Dauer keine Verbesserung in den Abläufen der Notfallstation erreicht werden kann.

Wechselnde Rahmenbedingungen liegen in der Natur jeder Notfallstation. Für unsere Arbeit bedeutete dies in den letzten Jahren konkret:

- Es zeigte sich ein ständiges Anwachsen der Patientenzahlen, insbesondere der sogenannten «Bagatellfälle» im ambulanten Bereich, verbunden mit einem Anstieg der Behandlungsdauer.

- In der Bevölkerung wie auch bei den Zuweisern machte sich eine zunehmende Unzufriedenheit breit.

- Es kam zu einer deutlichen Zunahme der ambulanten Nachkontrollen.

Unser interdisziplinäres Konzept beruht auf drei Eckpfeilern:

1. Das Leitungsteam der Notfallstation besteht aus einem vollamtlichen Ärztlichen Leiter sowie mehreren notfalleigenen Oberärzten. Dem Ärztlichen Leiter obliegt die organisatorische und fachliche Leitung der Notfallstation.

2. Die Rotationsassistenten der Medizinischen und der Chirurgischen Klinik «gehören» während der Rotation der Notfallstation und arbeiten interdisziplinär.

3. Einführung einer Kurzzeitbehandlungsspur («fast track»).

\section{Zu Punkt 1}

Grundlegender Gedanke ist die ständige Präsenz eines Kaderarztes auf der Notfallstation. Dieser «Teamleader» muss in der Lage sein, alle Notfälle aller Fachrichtungen beurteilen und behandeln zu können. Dies setzt eine breite Ausbildung, idealerweise als Internist oder Allgemeinmediziner mit breiter Erfahrung in Chirurgie, voraus. Erfahrung in Abdominalsonographie und ATLS/ACLS sind wünschenswert. Unsere Teamleader arbeiten werktags in zwei Schichten (7.30-16.30 Uhr und 15.00-23.00 Uhr) und an Wochenenden in einer Schicht (8.45-20.45 Uhr). In dieser Zeit wird jeder Patient, unabhängig von der Schwere oder Art seiner Erkrankung oder Verletzung, vom Teamleader bei Bedarf sowie auf jeden Fall abschliessend persönlich beurteilt und einer ambulanten oder stationären Behandlung zugeführt. Diese Arbeit umfasst weit mehr als nur eine Triagefunktion, vielmehr ersetzt der Teamleader des Notfalls die bisherige Funktion der Hintergrunddienste der einzelnen Kliniken, das heisst, er ist vollumfänglich für das gesamte Management des Patienten verantwortlich, je nach Situation in Zusammenarbeit mit den Hintergrunddiensten der verschiedenen Kliniken. Der Informationsfluss ins Spital wird durch tägliche Rapporte der einzelnen Kliniken und persönliche Übergaben gewährleistet.

Während der Abwesenheiten des Teamleaders (also nachts) wird die Notfallversorgung auf Kaderarztebene wie bisher durch die Hintergrunddienste der einzelnen Kliniken gewährleistet.

\section{Zu Punkt 2}

Der Assistentenpool der Notfallstation umfasst je fünf Rotationsassistenten der Medizinischen und Chirurgischen Klinik, welche zwischen drei und neun Monaten bei uns tätig sind. Die Assistenten arbeiten in drei Schichten (zwei Assistenzärzte Frühdienst 7.30-17.30 Uhr, zwei Assistenzärzte Spätdienst 13.00-23.00 Uhr, zwei Nachtärzte 22.30-7.45 Uhr). Im Gegensatz zu bisherigen Notfallsystemen arbeiten die Assistenzärzte zu den Präsenzzeiten des Teamleaders vollumfänglich interdisziplinär. Dies bedeutet konkret, dass chirurgische Assistenten auch medizinische Notfälle betreuen und umgekehrt, dies immer unter Aufsicht des Teamleaders. Dies erlaubt eine flexiblere Handhabung der personellen Ressourcen auf ärztlicher Ebene. Bei Abwesenheit des Teamleaders wird die Notfallstation mit einem chirurgischen und einem medizinischen Nachtarzt und dementsprechender Betreuung durch die Hintergrunddienste betrieben.

\section{Zu Punkt 3}

Ein Grossteil unserer Patienten bedarf nicht der Infrastruktur eines Spitals, sondern kommt wegen sogenannter Bagatellverletzungen oder Bagatellkrankheiten. Diese Patienten werden in zwei separaten Kurzzeitbehandlungszimmern («fast track») versorgt, mit dem Ziel, unser Haus innerhalb von 90 Minuten wieder zu verlassen. So bleibt zusätzliche räumliche und personelle Kapazität, um sich auf die spitalpflichtigen Patienten zu konzentrieren. Hier muss ganz klar betont werden, dass wir keine Permanence betreiben und nicht aktiv Patienten rekrutieren, dies würde dem Grundgedanken der «fast track»Spur fundamental widersprechen.

Am 10. Januar 2005 konnten wir mit diesem interdisziplinären Konzept unsere Arbeit aufnehmen - Grund genug, nach einem Jahr die ersten Erfahrungen wiederzugeben.

\section{Ergebnisse}

2005 hatten wir auf unserer Notfallstation 12276 ambulante und 3501 stationäre Konsultationen aller Fachrichtungen und aller Altersklassen. Im 
Abbildung 1

Konsultationen der Notfallstation und des Ambulatoriums 1997-2005.

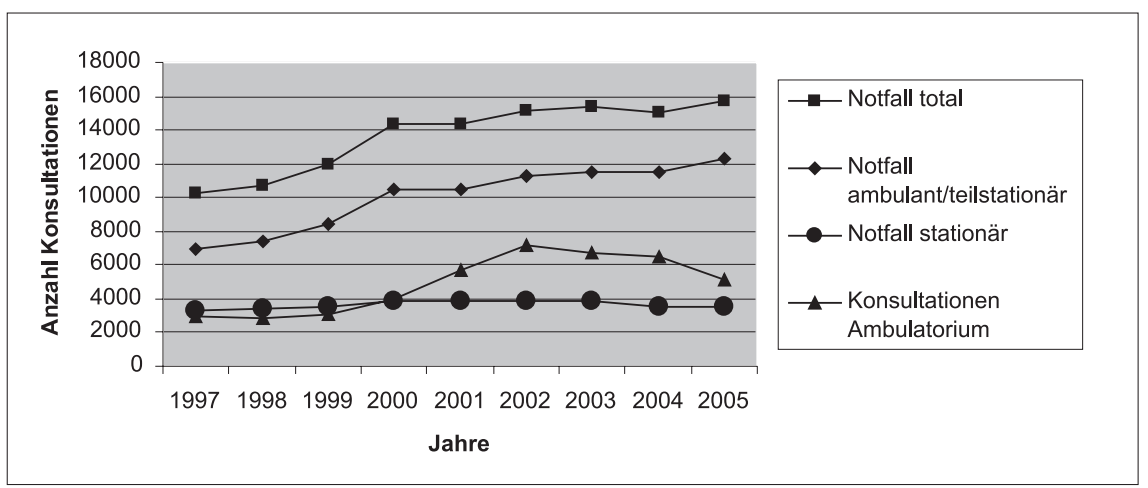

\section{Abbildung 2}

Vergleich Anzahl Notfallkonsultationen versus durchschnittliche Aufenthaltsdauer auf der Notfallstation 1997-2005.

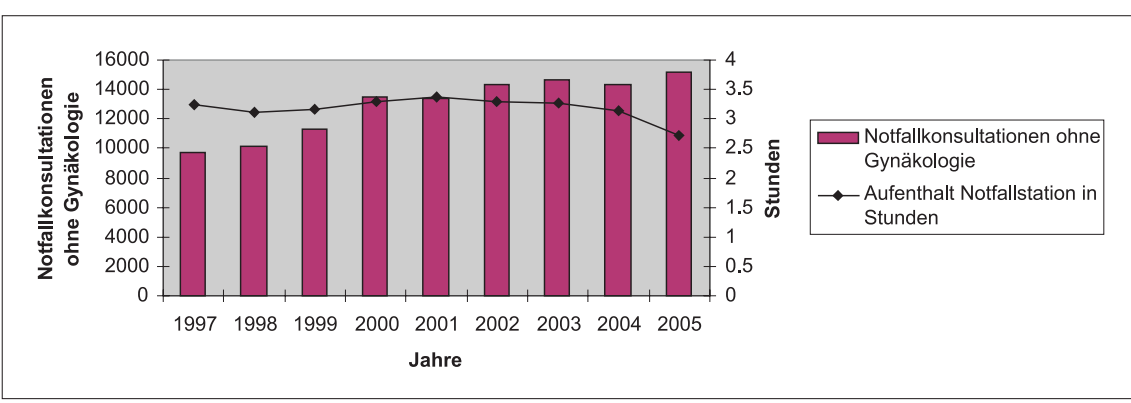

\section{Abbildung $3 a$}

Vergleich Anzahl Notfallkonsultationen versus durchschnittliche Aufenthaltsdauer auf der Notfallstation 1997-2005 bei chirurgischen Patienten.
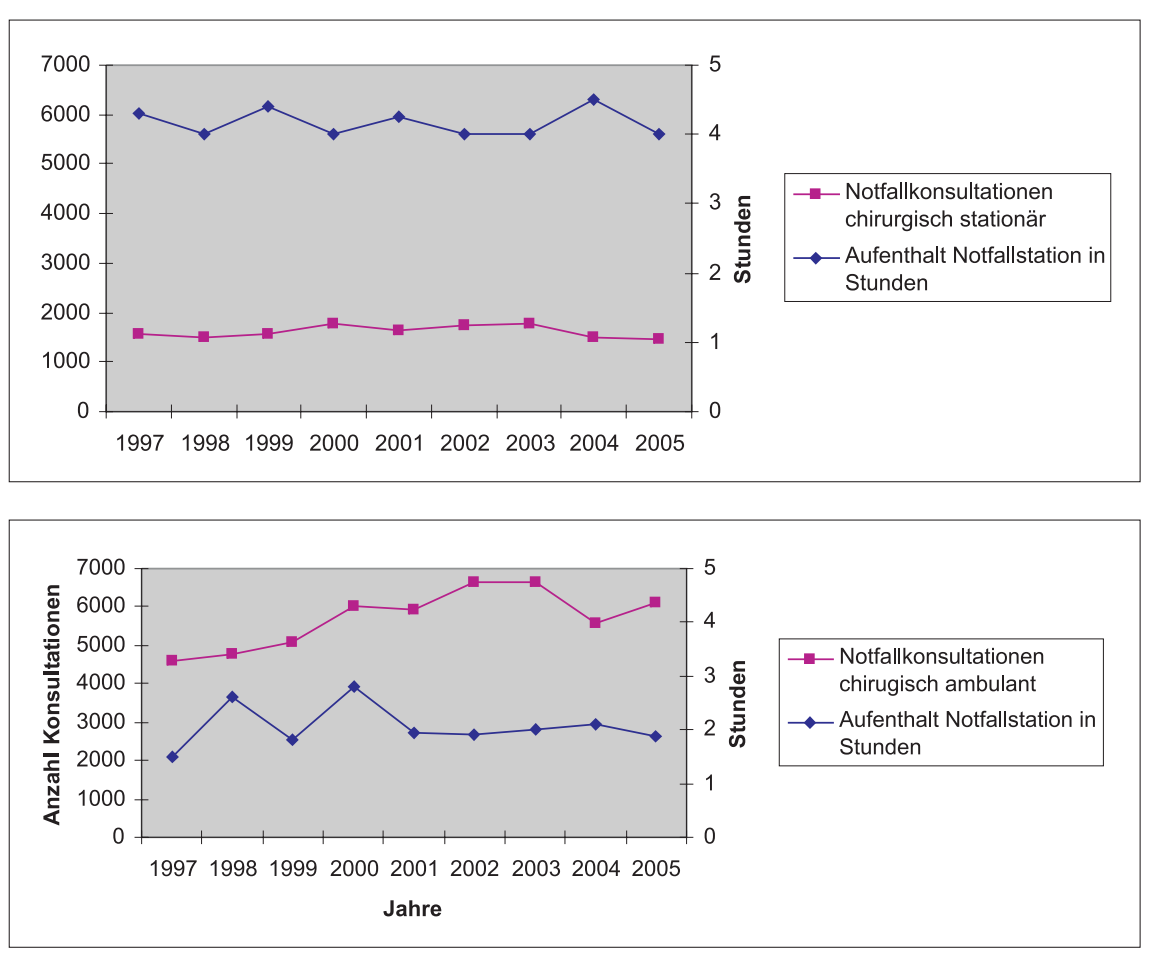

angegliederten Ambulatorium mit Gipszimmer wurden 20055142 Konsultationen durchgeführt. Gynäkologische und geburtshilfliche Notfälle werden durch die Frauenklinik versorgt, teils aber unter Benützung der Infrastruktur der Notfallstation.

Trotz stetig ansteigender Notfallkonsultationen (Abb. 1) konnte die Gesamtaufenthaltsdauer auf der Notfallstation im Vergleich zu den Vorjahren gesenkt werden (Abb. 2), je nach Fachrichtung mehr oder weniger stark ausgeprägt (Abb. 3a-c), obwohl wir während rund dreier Monate infolge Umbaus auf einer Baustelle arbeiteten und während rund zweier Monate nur vier statt neun Kojen zur Verfügung hatten!

Es zeigte sich ein deutlicher Rückgang der Nachkontrollen (-20,4\% im Vergleich 2004/ 2005) in unserem Ambulatorium (Abb. 1), bedingt durch ein rigoroses Zurückschicken der Patienten an die Hausärzte. Eine Nachkontrolle bei uns erfolgte nur in speziellen Situationen oder auf Wunsch des Patienten oder Hausarztes. Diese «Rückweisungspraxis» konnte auch an einer von uns durchgeführten Zuweiserbefragung bestätigt werden.

Radiologische Untersuchungen wurden reduziert (Abb. 4a), teils durch weniger häufige Nachkontrollen, aber auch durch gezieltere und kritischere Indikationsstellung. Dies beweist der Rückgang der radiologischen Untersuchungen trotz Zunahme der Notfallkonsultationen (Abb. 4a-c).

Ebenfalls konnte die Anzahl der Laboranalysen deutlich gesenkt werden. Es zeigte sich zwar - bei steigendem Patientenaufkommen - eine leichte Zunahme der Laboraufträge, aber eine deutliche Verminderung der Analysen (-8614). Dies entspricht einer Verringerung von $-9,1 \%$ (Abb. 5). Dies spricht für eine gezieltere Indikationsstellung.

Die Kosten an Medikamenten und Verbrauchsmaterial konnten pro Patient um 7,5\% gesenkt werden (Abb. 6), ein deutlicher Unterschied im Vergleich zur Vorperiode.

Im Bereiche der ambulanten Notfallpatienten kam es zu einer Senkung der Kosten/Austritt um 11,25\%, dies bei einem gleichzeitigen Anstieg der Kostendeckung um 3,8\% auf 91,5\% (Abb. 7) im Vergleich zur entsprechenden Vorjahresperiode. Auch das sind Hinweise auf ein effizienteres und kostenbewussteres Arbeiten durch die ständige Präsenz eines Kaderarztes auf der Notfallstation.

\section{Diskussion}

Im Zentrum der klinischen Notfallmedizin muss eine schnelle und kompetente Beurteilung des Patienten stehen mit dem Ziel, die aktuelle gesundheitliche Bedrohung des Patienten zu 
Abbildung $\mathbf{3 b}$

Vergleich Anzahl Notfallkonsultationen versus durchschnittliche Aufenthaltsdauer auf der Notfallstation 1997-2005 bei internistischen Patienten.
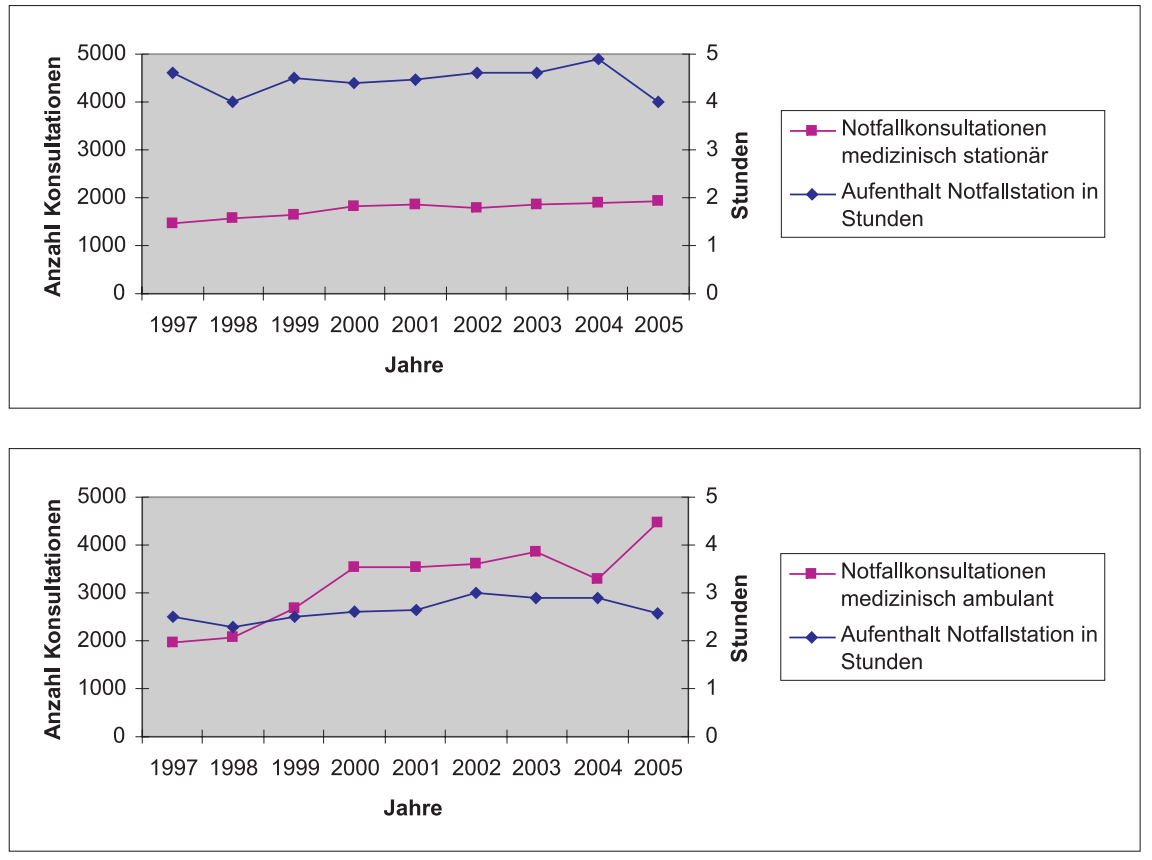

Abbildung 3c

Vergleich Anzahl Notfallkonsultationen versus durchschnittliche Aufenthaltsdauer auf der Notfallstation 1997-2005 bei urologischen Patienten.
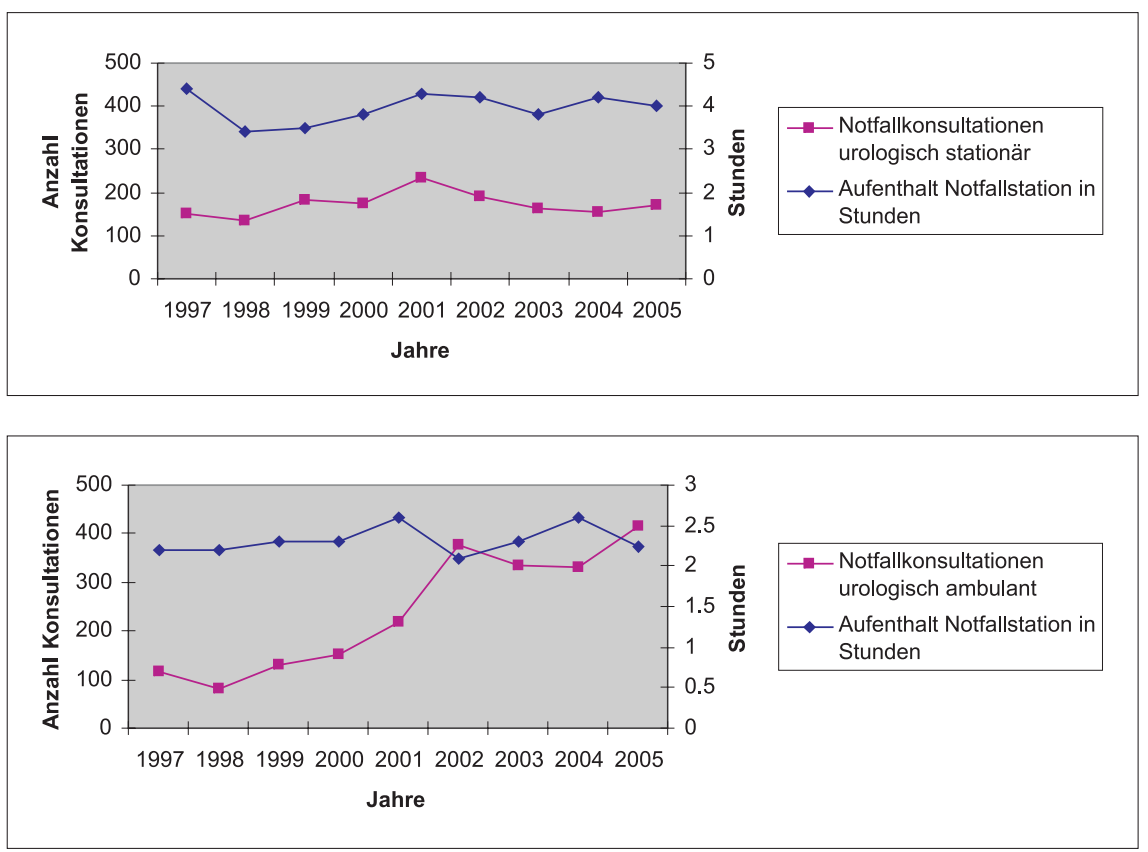

erkennen und zu behandeln. Ein sinnvoller Einsatz der damit verbundenen materiellen und personellen Ressourcen ist unabdingbar, eine ständige Kaderarztpräsenz wünschenswert. Dass dieses Konzept funktioniert, beweist die tägliche Arbeit auf Notfallstationen vor allem im englischsprachigen Raum.

Das interdisziplinäre Notfallkonzept des Gesundheitszentrums Zürich West ist unseres Wissens ein Novum in der klinischen Notfallmedizin bei Erwachsenen in der Schweiz. Das Konzept der Interdisziplinarität unter eigenständiger Leitung wurde bereits im April 2002 in der Notfallstation der Universitätskinderklinik Zürich eingeführt. Dabei zeigten sich die gleichen Ergebnisse, wie sie nachher auch bei uns beobachtet wurden: Verbesserte Ausbildung, Fokussierung auf klinische Untersuchung, Verzicht auf unnötige Untersuchungen und Kostenersparnis [1]. Unseres Erachtens bietet dieses Konzept nur Vorteile:

- Die ständige Mitarbeit eines interdisziplinär arbeitenden Kaderarztes auch zu unattraktiven Zeiten hat einen hohen Ausbildungsund Teachingeffekt für die Assistenzärzte. Sie werden sensibilisiert für Notfälle jeder Art und lernen, über den Tellerrand ihres eigentlichen Fachgebietes hinauszuschauen, ohne dass es zu einer Aushöhlung des Wissensstandes kommt.

- Die ständige Anwesenheit des Teamleaders gewährleistet auch bei kritischen Situationen ein schnelles Eingreifen.

- Durch die konsequente Beurteilung jedes Patienten durch einen Kaderarzt können relevante gesundheitliche Beeinträchtigungen frühzeitig erkannt werden.

- Mit der Einführung einer «fast track»-Spur kann einer Überlastung der Notfallstation durch nichtspitalpflichtige Notfälle entgegengewirkt werden.

- Für den Patienten unnötige Wartezeiten werden auf ein Minimum reduziert.

- Durch engen Einbezug der nachbehandelnden Kliniken kommt es zu keinem Informationsverlust in der Nachbehandlung. Die klinikeigenen Hintergrunddienste werden entlastet und können sich vermehrt ihren Kernaufgaben widmen.

- Durch gezielte Indikationsstellungen können radiologische und laborchemische Untersuchungen verringert werden.

- Einer weiteren Kostenexplosion kann - zumindest partiell - entgegengewirkt werden. 
Abbildung 4a

Vergleich aller radiologischen Untersuchungen der Notfallstation und des Ambulatoriums 2004 und 2005.

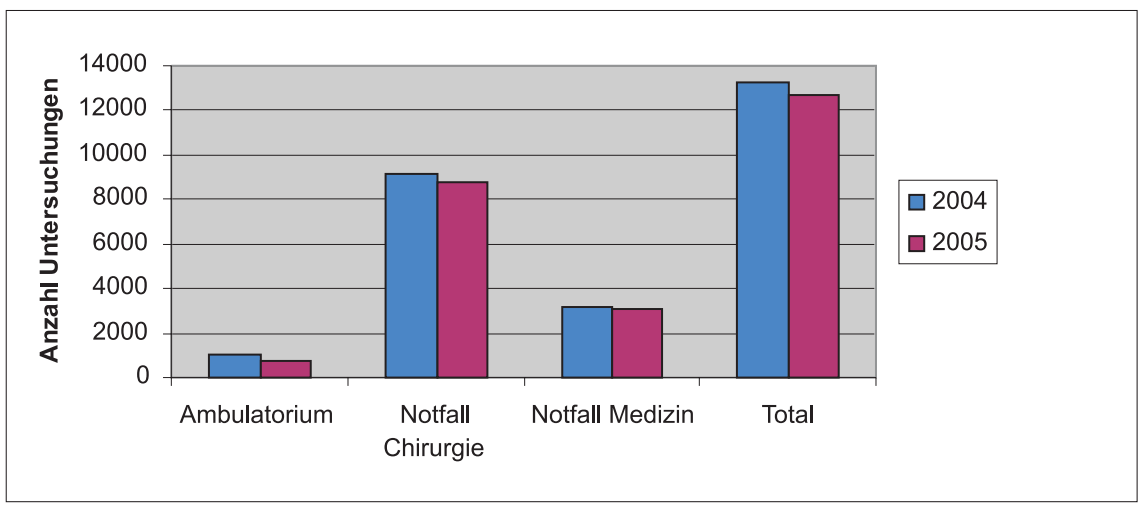

Abbildung 4b

Vergleich aller radiologischen Untersuchungen der chirurgischen Notfälle 2004 und 2005.

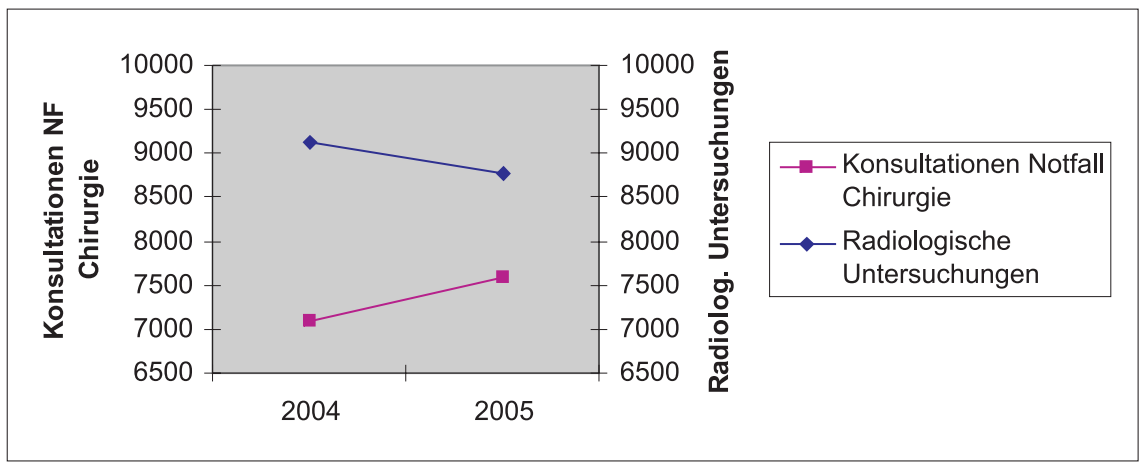

Abbildung 4c

Vergleich aller radiologischen Untersuchungen der medizinischen Notfälle 2004 und 2005.

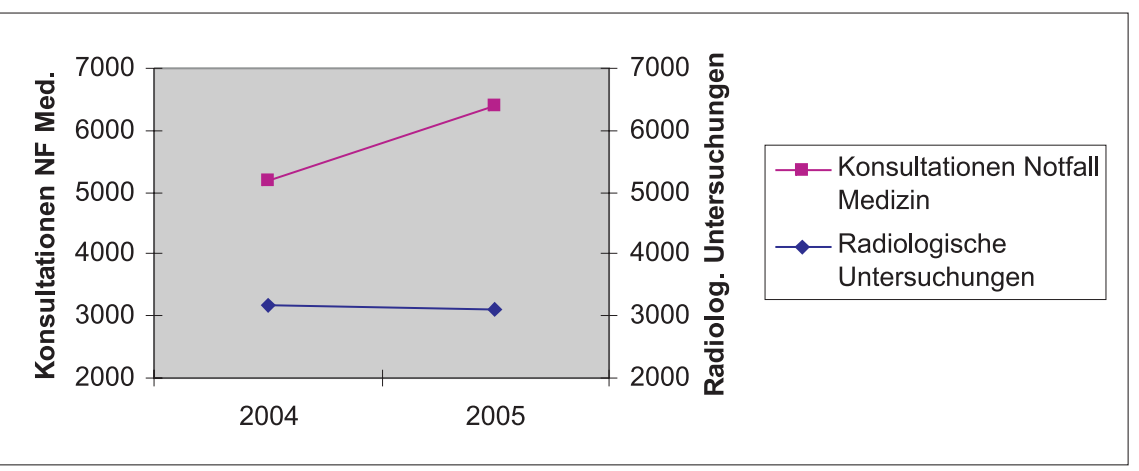

\section{Interdisziplinarität - was soll das?}

Aufgrund unser Erfahrung im Jahr 2005 und der hier gezeigten Ergebnisse dürfte der Vorteil einer selbständig und wirklich interdisziplinär geführten Notfallstation unbestritten sein. Die ständige Präsenz eines Teamleaders, welcher konsequent mit dem Assistententeam alle Patienten behandelt, bringt nur Vorteile.

Die Interdisziplinarität auf Assistentenebene birgt ein hohes Potential in der Ausbildung des Ärztenachwuchses: Schon früh werden die Assistenten für eine ganzheitliche Sicht des Patienten sensibilisiert und lernen, mit Notfällen jedwelcher Art umzugehen, dies unabhängig von der jeweiligen Ausbildungsrichtung. Der Blick über den Gartenzaun des eigenen Fachgebietes ist interessant, fördert das Verständnis der Arbeit anderer Fachdisziplinen und führt $\mathrm{zu}$ einem flexibleren Denken im Alltag. Nicht zuletzt wird durch die Interdisziplinarität auf Assistentenebene (wie auch auf Kaderarztebene) ein gut zusammenarbeitendes Team geschaffen, welches auch in schwierigen Momenten zusammenhält.

Durch die Schaffung des Fähigkeitsausweises «Klinische Notfallmedizin», welcher von der FMH und der SGNOR anerkannt wird, werden die Weichen in der Ausbildung zur klinischen Notfallmedizin richtig gestellt. Für die Zukunft wünschen wir uns weitere echte interdisziplinäre Notfallstationen mit eigenständiger Leitung und interdisziplinär arbeitenden Assistenten im Sinne der amerikanischen «emergency rooms». Dies bedingt ein Aufbrechen bestehender Strukturen und - zugegebenermassen - auch einen gewissen Verlust von Einflussnahme der involvierten Kliniken. Wir hoffen, dass in Zukunft weitere Chefärzte und Spitalleitungen diesen Mut aufbringen. Von einer engen, respektvollen und partnerschaftlichen Zusammenarbeit zwischen einer eigenständigen Notfallstation und dem Rest des Spitals profitieren alle: Das Gesamtspital, die einzelnen Kliniken und vor allem die uns anvertrauten Patienten, deren Wohl in all unserem Tun und Handeln unser oberstes Ziel sein sollte.

Literatur

1 Stocker S, Staubli G, Peier K, Tomaske M. Change of management - impact on the work in a paediatric emergency department. Swiss Med Wkly 2004;134(Suppl 141):S 15 
Abbildung 5

Vergleich laborchemischer Analysen und Aufträge versus Konsultationen 2004 und 2005.

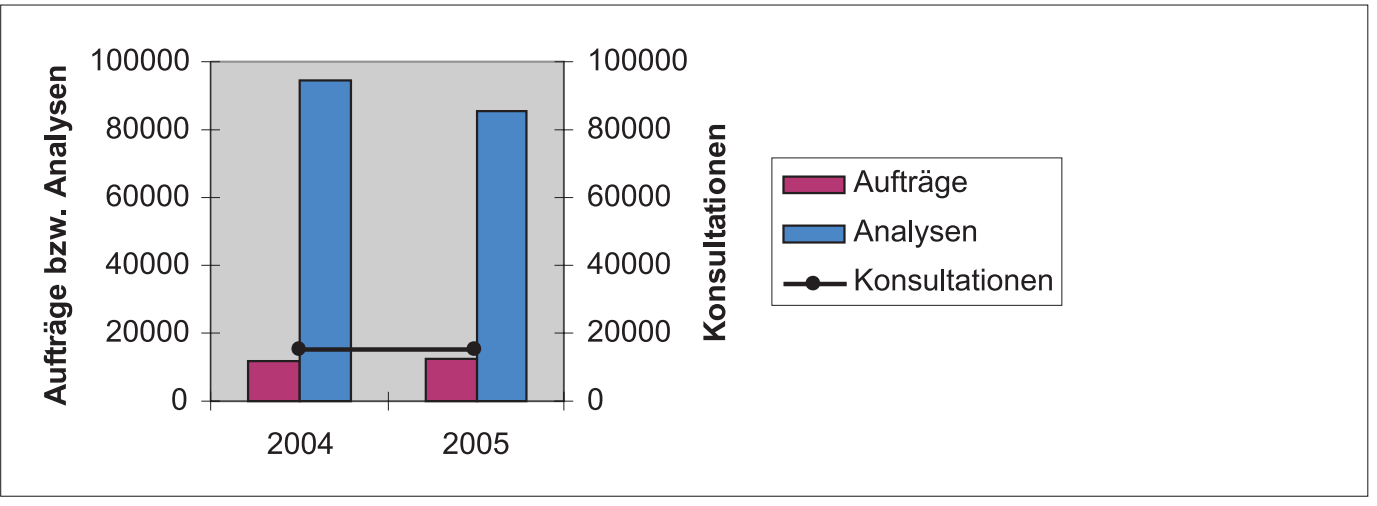

Abbildung 6

Vergleich Kosten Medikamente und Verbandsmaterial pro Patient 2003-2005.

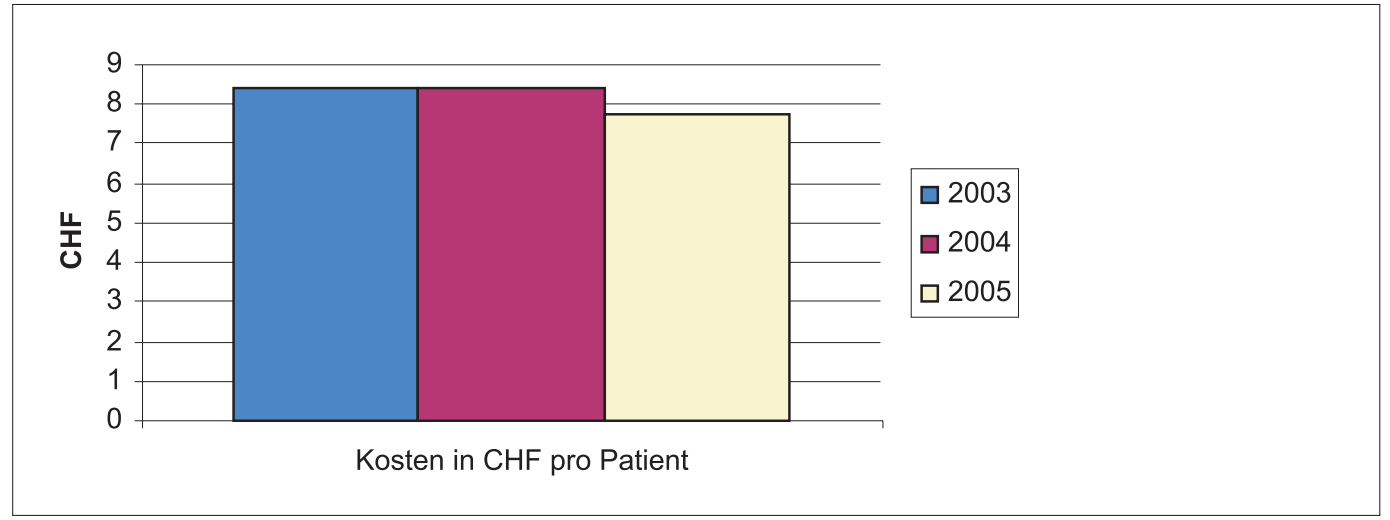

Abbildung 7

Vergleich Gesamtkosten und Kostendeckungsgrad pro ambulanter Austritt im 1.-3. Quartal 2004 und 2005.

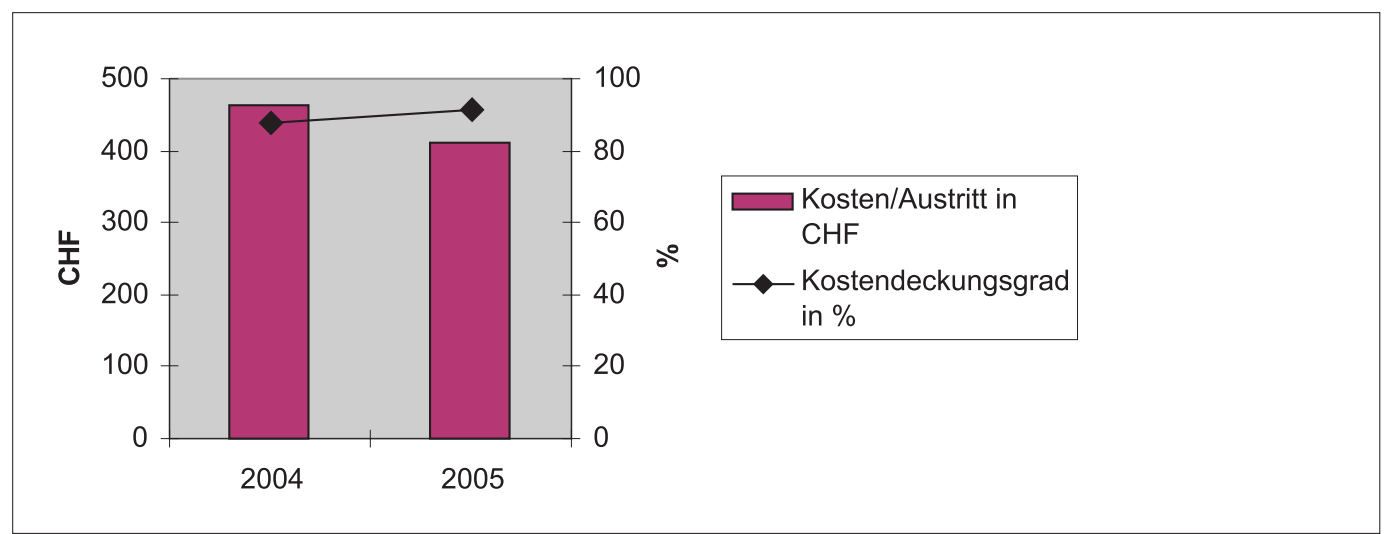

